International Journal of Pure and Applied Mathematics

Volume 92 No. 2 2014, 181-190

ISSN: 1311-8080 (printed version); ISSN: 1314-3395 (on-line version)

url: http://www.ijpam.eu

doi: http://dx.doi.org/10.12732/ijpam.v92i2.4

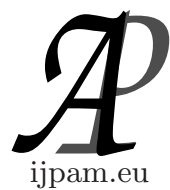

\title{
A SYSTEMATIC APPROACH FOR SOLVING MIXED INTUITIONISTIC FUZZY TRANSPORTATION PROBLEMS
}

\author{
P. Senthil Kumar ${ }^{1}$, R. Jahir Hussain ${ }^{2}$ \\ ${ }^{1,2} \mathrm{PG}$ and Research Department of Mathematics \\ Jamal Mohamed College (Autonomous) \\ Tirchirappalli, 620 020, Tamilnadu, INDIA
}

\begin{abstract}
In this paper, we formulate a transportation problem in which supplies, demands and costs are different types of real, fuzzy and intuitionistic fuzzy numbers. The new multiplication operations of triangular intuitionistic fuzzy numbers are employed to get the optimal solution. Mixed Intuitionistic fuzzy zero point method is proposed to find the optimal solution in terms of triangular intuitionistic fuzzy numbers. The proposed method is easy to understand and to apply for finding the intuitionistic fuzzy optimal solution in a mixed intuitionistic fuzzy transportation problem. The method is illustrated by a numerical example.
\end{abstract}

AMS Subject Classification: 03E72, 03F55, 90B06

Key Words: triangular intuitionistic fuzzy numbers, mixed intuitionistic fuzzy transportation problem, intuitionistic fuzzy zero point method, optimal solution

\section{Introduction}

The theory of fuzzy set introduced by L.A. Zadeh[11] in 1965 has achieved successful applications in various fields. The concept of Intuitionistic Fuzzy

Received: November 19, 2013

(c) 2014 Academic Publications, Ltd.

$\S$ Correspondence author url: www.acadpubl.eu 
Sets (IFSs) proposed by K.T.Atanassov[1] in 1986 is found to be highly useful to deal with vagueness. The concept of fuzzy mathematical programming was introduced by Tanaka et al in 1947 the frame work of fuzzy decision of Bellman and Zadeh[4].

In [2], Amit Kumar et al investigated fuzzy linear programming approach for solving fuzzy transportation problems with transshipment. In [9], P. Pandian and G. Natarajan presented a new algorithm for finding a fuzzy optimal solution for fuzzy transportation problem. In [10], D. Stephen Dinager et al presented Transportation problem in fuzzy environment. In [8],R. Jahir Hussain et all presented transportation problem with the aid of triangular intuitionistic fuzzy numbers. In [5], S. Ismail Mohideen and P. Senthil Kumar investigated a comparative study on transportation problem in fuzzy environment.

There is no systematic approach for finding an optimal solution for mixed intuitionistic fuzzy transportation problem, but when we search our literature it has demonstrated intuitionistic fuzzy transportation problems $[6,7,8]$ only. In this paper, a new ranking procedure which can be found in [3] and is used to obtain an optimal solution for a mixed intuitionistic fuzzy transportation problem [MIFTP].

This paper is organized as follows: In Section 2, some definitions and new multiplication operation is reviewed, In Section 3, provides the definition of intuitionistic fuzzy transportation problem and its mathematical formulation, In Section 4, solution procedure for solving mixed intuitionistic fuzzy transportation problem is discussed. In Section 5, to illustrate the proposed method a numerical example with results and discussion is discussed and followed by the conclusions are given in Section 6.

\section{Preliminaries}

Definition 1. Let $A$ be a classical set, $\mu_{A}(x)$ be a function from $A$ to $[0,1]$. A fuzzy set $A^{*}$ with the membership function $\mu_{A}(x)$ is defined by

$$
A^{*}=\left\{\left(x, \mu_{A}(x)\right) ; x \in A \text { and } \mu_{A}(x) \in[0,1]\right\} .
$$

Definition 2. Let $X$ be denote a universe of discourse, then an intuitionistic fuzzy set $A$ in $X$ is given by a set of ordered triples,

$$
\tilde{A}^{I}=\left\{<x, \mu_{A}(x), \nu_{A}(x)>; x \in X\right\}
$$

Where $\mu_{A}, \nu_{A}: X \rightarrow[0,1]$, are functions such that $0 \leq \mu_{A}(x)+\nu_{A}(x) \leq$ $1, \forall x \in X$. For each $\mathrm{x}$ the membership $\mu_{A}(x)$ and $\nu_{A}(x)$ represent the degree of 
membership and the degree of non-membership of the element $x \in X$ to $A \subset X$ respectively.

Definition 3. A fuzzy number $A$ is defined to be a triangular fuzzy number if its membership function $\mu_{A}: \mathbb{R} \rightarrow[0,1]$ is equal to

$$
\mu_{A}(x)= \begin{cases}\frac{\left(x-a_{1}\right)}{\left(a_{2}-a_{1}\right)} & \text { if } x \in\left[a_{1}, a_{2}\right] \\ \frac{\left(a_{3}-x\right)}{\left(a_{3}-a_{2}\right)} & \text { if } x \in\left[a_{2}, a_{3}\right] \\ 0 & \text { otherwise }\end{cases}
$$

where $a_{1} \leq a_{2} \leq a_{3}$. This fuzzy number is denoted by $\left(a_{1}, a_{2}, a_{3}\right)$.

Definition 4. A Triangular Intuitionistic Fuzzy Number $\left(\tilde{A}^{I}\right.$ is an intuitionistic fuzzy set in $\mathrm{R}$ with the following membership function $\mu_{A}(x)$ and non-membership function $\nu_{A}(x):$ )

$$
\mu_{A}(x)=\left\{\begin{array}{ll}
0 & \text { for } x<a_{1} \\
\frac{\left(x-a_{1}\right)}{\left(a_{2}-a_{1}\right)} & \text { for } a_{1} \leq x \leq a_{2} \\
1 & \text { for } x=a_{2} \\
\frac{\left(a_{3}-x\right)}{\left(a_{3}-a_{2}\right)} & \text { for } a_{2} \leq x \leq a_{3} \\
0 & \text { for } x>a_{3}
\end{array} \quad \nu_{A}(x)= \begin{cases}1 & \text { for } x<a_{1}^{\prime} \\
\frac{\left(a_{2}-x\right)}{\left(a_{2}-a_{1}^{\prime}\right)} & \text { for } a_{1}^{\prime} \leq x \leq a_{2} \\
0 & \text { for } x=a_{2} \\
\frac{\left(x-a_{2}\right)}{\left(a_{3}^{\prime}-a_{2}\right)} & \text { for } a_{2} \leq x \leq a_{3}^{\prime} \\
1 & \text { for } x>a_{3}^{\prime}\end{cases}\right.
$$

Where $a_{1}^{\prime} \leq a_{1} \leq a_{2} \leq a_{3} \leq a_{3}^{\prime}$ and $\mu_{A}(x), \nu_{A}(x) \leq 0.5$ for $\mu_{A}(x)=$ $\nu_{A}(x), \forall x \in \mathbb{R}$ This TrIFN is denoted by $\tilde{A}^{I}=\left(a_{1}, a_{2}, a_{3}\right)\left(a_{1}^{\prime}, a_{2}, a_{3}^{\prime}\right)$.

Particular Cases: Let $\tilde{A}^{I}=\left(a_{1}, a_{2}, a_{3}\right)\left(a_{1}^{\prime}, a_{2}, a_{3}^{\prime}\right)$ be a TrIFN. Then the following cases arise

Case 1: If $a_{1}^{\prime}=a_{1}, a_{3}^{\prime}=a_{3}$, then $\tilde{A}^{I}$ represent Tringular Fuzzy Number (TFN). It is denoted by $A=\left(a_{1}, a_{2}, a_{3}\right)$.

Case 2: If $a_{1}^{\prime}=a_{1}=a_{2}=a_{3}=a_{3}^{\prime}=m$, then $\tilde{A}^{I}$ represent a real number $m$.

\section{Ranking of triangular intuitionistic fuzzy numbers}

The Ranking of a triangular intuitionistic fuzzy number $\tilde{A}^{I}=\left(a_{1}, a_{2}, a_{3}\right)\left(a_{1}^{\prime}, a_{2}, a_{3}^{\prime}\right)$ is defined by

$$
R\left(\tilde{A}^{I}\right)=\frac{1}{3}\left[\frac{\left(a_{3}^{\prime}-a_{1}^{\prime}\right)\left(a_{2}-2 a_{3}^{\prime}-2 a_{1}^{\prime}\right)+\left(a_{3}-a_{1}\right)\left(a_{1}+a_{2}+a_{3}\right)+3\left(a_{3}^{\prime 2}-a_{1}^{\prime 2}\right)}{a_{3}^{\prime}-a_{1}^{\prime}+a_{3}-a_{1}}\right]
$$

If $\nu_{A}(x)=1-\mu_{A}(x)$, then $\operatorname{TrIFN} \tilde{A}^{I}=\left(a_{1}, a_{2}, a_{3}\right)\left(a_{1}^{\prime}, a_{2}, a_{3}^{\prime}\right)$ will become the $\operatorname{TrFN}\left(a_{1}, a_{2}, a_{3}\right)$. Then $a_{1}^{\prime}=a_{1}$ and $a_{3}^{\prime}=a_{3}$

$$
R\left(\tilde{A}^{I}\right)=\left(a_{1}+a_{2}+a_{3}\right) / 3
$$


The Rank of TrIFN $\tilde{A}^{I}=\left(a_{1}, a_{2}, a_{3}\right)\left(a_{1}^{\prime}, a_{2}, a_{3}^{\prime}\right)$ is defined by $R\left(\tilde{A}^{I}\right)=a_{2}$ if $a_{2}-$ $a_{1}=a_{3}-a_{2}$ and $a_{2}-a_{1}^{\prime}=a_{3}^{\prime}-a_{2}$. Let $\tilde{A}^{I}=(8,10,12)(6,10,14)$ be a TrIFN, then its rank is defined by $R\left(\tilde{A}^{I}\right)=10$.

Definition 5. Let $\tilde{A}^{I}$ and $\tilde{B}^{I}$ be two TrIFNs. The ranking of $\tilde{A}^{I}$ and $\tilde{B}^{I}$ by the $R($.$) on E$, the set of TrIFNs is defined as follows:

(i) $R\left(\tilde{A}^{I}\right)>R\left(\tilde{B}^{I}\right)$ iff $\tilde{A}^{I} \succ \tilde{B}^{I}$,

(ii) $R\left(\tilde{A}^{I}\right)<R\left(\tilde{B}^{I}\right)$ iff $\tilde{A}^{I} \prec \tilde{B}^{I}$,

(iii) $R\left(\tilde{A}^{I}\right)=R\left(\tilde{B}^{I}\right)$ iff $\tilde{A}^{I} \approx \tilde{B}^{I}$

(iv) $R\left(\tilde{A}^{I}+\tilde{B}^{I}\right)=R\left(\tilde{A}^{I}\right)+R\left(\tilde{B}^{I}\right)$,

(v) $R\left(\tilde{A}^{I}-\tilde{B}^{I}\right)=R\left(\tilde{A}^{I}\right)-R\left(\tilde{B}^{I}\right)$,

(vi) $R\left(\tilde{A}^{I} \otimes \tilde{B}^{I}\right)=R\left(\tilde{A}^{I}\right) \otimes R\left(\tilde{B}^{I}\right)$.

Definition 6. The ordering $\succcurlyeq$ and $\preccurlyeq$ between any two TrIFNs $\tilde{A}^{I}$ and $B^{I}$ are defined as follows:

(i) $\tilde{A}^{I} \succcurlyeq \tilde{B}^{I}$ iff $\tilde{A}^{I} \succ \tilde{B}^{I}$ or $\tilde{A}^{I} \approx \tilde{B}^{I}$, (ii) $\tilde{A}^{I} \preccurlyeq \tilde{B}^{I}$ iff $\tilde{A}^{I} \prec \tilde{B}^{I}$ or $\tilde{A}^{I} \approx \tilde{B}^{I}$.

\section{Arithmetic Operations :}

Let $\tilde{A}^{I}=\left(a_{1}, a_{2}, a_{3}\right)\left(a_{1}^{\prime}, a_{2}, a_{3}^{\prime}\right)$ and $\tilde{B}^{I}=\left(b_{1}, b_{2}, b_{3}\right)\left(b_{1}^{\prime}, b_{2}, b_{3}^{\prime}\right)$ be any two TrIFNs then the arithmetic operations as follows.

Addition: $\tilde{A}^{I} \oplus \tilde{B}^{I}=\left(a_{1}+b_{1}, a_{2}+b_{2}, a_{3}+b_{3}\right)\left(a_{1}^{\prime}+b_{1}^{\prime}, a_{2}+b_{2}, a_{3}^{\prime}+b_{3}^{\prime}\right)$.

Subtraction: $\tilde{A}^{I} \ominus \tilde{B}^{I}=\left(a_{1}-b_{3}, a_{2}-b_{2}, a_{3}-b_{1}\right)\left(a_{1}^{\prime}-b_{3}^{\prime}, a_{2}-b_{2}, a_{3}^{\prime}-b_{1}^{\prime}\right)$.

Multiplication: If $R\left(\tilde{A}^{I}\right), R\left(\tilde{B}^{I}\right) \geq 0$, then

$$
\begin{aligned}
\tilde{A}^{I} \otimes \tilde{B}^{I}=\left[\left(\frac{a_{1}\left(b_{1}+b_{2}+b_{3}\right)}{3}, \frac{a_{2}\left(b_{1}+b_{2}+b_{3}\right)^{\prime}}{3} \frac{a_{3}\left(b_{1}+b_{2}+b_{3}\right)}{3}\right)\right. \\
\left.\left(\frac{a_{1}^{\prime}\left(b_{1}^{\prime}+b_{2}+b_{3}^{\prime}\right)}{3}, \frac{a_{2}\left(b_{1}^{\prime}+b_{2}+b_{3}^{\prime}\right)}{3}, \frac{a_{3}^{\prime}\left(b_{1}^{\prime}+b_{2}+b_{3}^{\prime}\right)}{3}\right)\right] .
\end{aligned}
$$

If $R\left(\tilde{A}^{I}\right), R\left(\tilde{B}^{I}\right)<0$, then

$$
\begin{aligned}
\tilde{A}^{I} \otimes \tilde{B}^{I}= & {\left[\left(\frac{a_{3}\left(b_{1}+b_{2}+b_{3}\right)}{3}, \frac{a_{2}\left(b_{1}+b_{2}+b_{3}\right)^{\prime}}{3} \frac{a_{1}\left(b_{1}+b_{2}+b_{3}\right)}{3}\right)\right.} \\
& \left.\left(\frac{a_{3}^{\prime}\left(b_{1}^{\prime}+b_{2}+b_{3}^{\prime}\right)}{3}, \frac{a_{2}\left(b_{1}^{\prime}+b_{2}+b_{3}^{\prime}\right)}{3}, \frac{a_{1}^{\prime}\left(b_{1}^{\prime}+b_{2}+b_{3}^{\prime}\right)}{3}\right)\right] .
\end{aligned}
$$

Scalar Addition: $R\left(K+\tilde{A}^{I}\right)=K+R\left(\tilde{A}^{I}\right)$.

Scalar Multiplication:

i. $k \tilde{A}^{I}=\left(k a_{1}, k a_{2}, k a_{3}\right)\left(k a_{1}^{\prime}, k a_{2}, k a_{3}^{\prime}\right)$, for $k \geq 0$ 
ii. $k \tilde{A}^{I}=\left(k a_{3}, k a_{2}, k a_{1}\right)\left(k a_{3}^{\prime}, k a_{2}, k a_{1}^{\prime}\right)$, for $k<0$

\section{Intuitionistic Fuzzy Transportation Problem}

Consider a transportation with $\mathrm{m}$ origins (rows) and $\mathrm{n}$ destinations (columns). Let $\tilde{c}_{i j}^{I}$ be the cost of transporting one unit of the product from $i^{\text {th }}$ origin to $j^{\text {th }}$ destination. Let $\tilde{a}_{i}^{I}$ be the quantity of commodity available at origin $i$. Let $\tilde{b}_{j}^{I}$ be the quantity of commodity needed at destination $\mathrm{j}$ and $\tilde{x}_{i j}^{I}$ is the quantity transported from $i^{\text {th }}$ origin to $j^{\text {th }}$ destination, so as to minimize the Intuitionistic Fuzzy Transportation Cost [IFTC].

$$
\begin{aligned}
\text { (IFTP)Minimize } \tilde{Z}^{I}=\sum_{i=1}^{m} \sum_{j=1}^{n} \tilde{c}_{i j}^{I} \otimes \tilde{x}_{i j}^{I} \\
\text { Subject to, } \quad \begin{aligned}
& \sum_{j=1}^{n} \tilde{x}_{i j}^{I} \approx \tilde{a}_{i}^{I}, \quad \text { for } i=1,2, \cdots, m \\
& \sum_{i=1}^{m} \tilde{x}_{i j}^{I} \approx \tilde{b}_{j}^{I}, \quad \text { for } j=1,2, \cdots, n \\
& \tilde{x}_{i j}^{I} \approx \tilde{0}^{I}, \quad \text { for } i=1,2, \cdots, m \text { and } j=1,2, \cdots, n
\end{aligned}
\end{aligned}
$$

Where $m=$ the number of supply points, $n=$ the number of demand points $\tilde{c}_{i j}^{I}=\left(c_{i j}^{1}, c_{i j}^{2}, c_{i j}^{3}\right)\left(c_{i j}^{1^{\prime}}, c_{i j}^{2}, c_{i j}^{3^{\prime}}\right), \tilde{a}_{i}^{I}=\left(a_{i}^{1}, a_{i}^{2}, a_{i}^{3}\right)\left(a_{i}^{1^{\prime}}, a_{i}^{2}, a_{i}^{3^{\prime}}\right)$, $\tilde{b}_{j}^{I}=\left(b_{j}^{1}, b_{j}^{2}, b_{j}^{3}\right)\left(b_{j}^{1^{\prime}}, b_{j}^{2}, b_{j}^{3^{\prime}}\right), \tilde{x}_{i j}^{I}=\left(x_{i j}^{1}, x_{i j}^{2}, x_{i j}^{3}\right)\left(x_{i j}^{1^{\prime}}, x_{i j}^{2}, x_{i j}^{3^{\prime}}\right)$

\section{The Computational Procedure for Mixed Intuitionistic Fuzzy Transportation Problem}

Step 1. Construct the transportation table in which costs, supplies and demands are must be a combination of real, fuzzy and intuitionistic fuzzy numbers. Convert the given MIFTP into a balanced mixed intuitionistic fuzzy transportation problem(BMIFTP), if it is not, by ranking method. Step 2. After using step 1 convert BMIFTP into balanced intuitionistic fuzzy transportation problem (BIFTP) using the following steps

i. If any one or more in the supplies (demands and costs) of a transportation problem having a real number say $a_{1}$ that can be expanded as a TrIFN $a_{1}=\left(a_{1}, a_{1}, a_{1}\right)\left(a_{1}, a_{1}, a_{1}\right)$.

ii. If any one or more in the supplies (demands and costs) of a transportation problem having a triangular fuzzy number say $\left(a_{1}, a_{2}, a_{3}\right)$ that can be expanded as a $\operatorname{TrIFN}\left(a_{1}, a_{2}, a_{3}\right)=\left(a_{1}, a_{2}, a_{3}\right)\left(a_{1}, a_{2}, a_{3}\right)$. 
iii. If any one or more in the supplies (demands and costs) of a transportation problem having a TrIFN say $\left(a_{1}, a_{2}, a_{3}\right)\left(a_{1}^{\prime}, a_{2}, a_{3}^{\prime}\right)$ that can be kept as it is. That is $\left(a_{1}, a_{2}, a_{3}\right)\left(a_{1}^{\prime}, a_{2}, a_{3}^{\prime}\right)=\left(a_{1}, a_{2}, a_{3}\right)\left(a_{1}^{\prime}, a_{2}, a_{3}^{\prime}\right)$

Step 3. In the cost matrix subtract the smallest element in each row from every element of that row.

Step 4. In the reduced matrix that is after using the step 3, subtract the smallest element in each column from every element of that column.

Step 5. Check if each row intuitionistic fuzzy supply is less than or equal to sum of the column Intuitionistic fuzzy demands whose reduced costs in that row are intuitionistic fuzzy zero. Also, check if each column intuitionistic fuzzy demand is less than or equal to the sum of the intuitionistic fuzzy supplies whose reduced costs in that column are intuitionistic fuzzy zero. If so, go to step 8. Otherwise, go to step 6.

Step 6. Draw the minimum number of vertical lines and horizontal lines to cover all the intuitionistic fuzzy zeros of the reduced cost matrix such that some entries of row(s) or / and column(s) which do not satisfy the condition of the step 5 are not covered.

Step 7. Develop the new revised reduced cost matrix table as follows:

i. Select the smallest element among all the uncovered elements in the cost matrix.

ii. Subtract this least element from all the uncovered elements and add it to the element which lies at the intersection of any two lines. Thus, we obtain the modified cost matrix and then go to step 5 .

Step 8. Select a cell in the reduced cost matrix whose reduced cost is the maximum cost say $(\alpha, \beta)$. If there are more than one occur then select arbitrarily.

Step 9. Select a cell in the $\alpha$ - row or / and $\beta$ - column of the reduced cost matrix which is the only cell whose reduced cost is intuitionistic fuzzy zero and then allot the maximum possible value to that cell. If such cell does not occur for the maximum value, find the next maximum so that such a cell occurs. If such cell does not occur for any value, we select any cell in the reduced cost matrix whose reduced cost is intuitionistic fuzzy zero.

Step 10. Reform the reduced intuitionistic fuzzy transportation table after deleting the fully used intuitionistic fuzzy supply points and the fully received intuitionistic fuzzy demand points and also, modify it to include the not fully used intuitionistic fuzzy supply points and the not fully received intuitionistic fuzzy demand points. 
Step 11. Repeat step 8 to the step 10 until all intuitionistic fuzzy supply points are fully used and all intuitionistic fuzzy demand points are fully received. This allotment yields an optimal solution.

\section{Numerical Example}

Consider the $3 \times 3$ MIFTP

\begin{tabular}{|c|c|c|c|c|}
\hline & $D_{1}$ & $D_{2}$ & $D_{3}$ & Supply \\
\hline O1 & $(8,10,12)(6,10,14)$ & 4 & $(10,15,20)$ & $(4,6,8)(3,6,9)$ \\
\hline O2 & 3 & $(6,12,18)$ & $(4,6,8)(2,6,10)$ & 8 \\
\hline O3 & $(4,8,12)$ & $(3,4,5)(2,4,6)$ & 6 & $(2,5,8)$ \\
\hline Demand & $(3,4,5)$ & $(2,6,10)(1,6,11)$ & 9 & \\
\hline
\end{tabular}

The corresponding balanced intuitionistic fuzzy transportation table (BIFTT) is

\begin{tabular}{|c|c|c|c|c|}
\hline & $D_{1}$ & $D_{2}$ & $D_{3}$ & Supply \\
\hline O1 & $(8,10,12)(6,10,14)$ & $(4,4,4)(4,4,4)$ & $(10,15,20)(10,15,20)$ & $(4,6,8)(3,6,9)$ \\
\hline O2 & $(3,3,3)(3,3,3)$ & $(6,12,18)(6,12,18)$ & $(4,6,8)(2,6,10)$ & $(8,8,8)(8,8,8)$ \\
\hline O3 & $(4,8,12)(4,8,12)$ & $(3,4,5)(2,4,6)$ & $(6,6,6)(6,6,6)$ & $(2,5,8)(2,5,8)$ \\
\hline Demand & $(3,4,5)(3,4,5)$ & $(2,6,10)(1,6,11)$ & $(9,9,9)(9,9,9)$ & $(14,19,24)(13,19,25)$ \\
\hline
\end{tabular}

Since $\sum_{i=1}^{m} \tilde{a}_{i}^{I}=\sum_{j=1}^{n} \tilde{b}_{j}^{I}=(14,19,24)(13,19,25)$, the problem is BIFTP.

Now, using the step 3 to the step 4 of the intuitionistic fuzzy zero point method, we have the following reduced intuitionistic fuzzy transportation table.

\begin{tabular}{|c|c|c|c|c|}
\hline & $D_{1}$ & $D_{2}$ & $D_{3}$ & Supply \\
\hline O1 & $(4,6,8)(2,6,10)$ & $(0,0,0)(0,0,0)$ & $(3,9,15)(2,9,16)$ & $(4,6,8)(3,6,9)$ \\
\hline O2 & $(0,0,0)(0,0,0)$ & $(3,9,15)(3,9,15)$ & $(-2,1,4)(-5,1,7)$ & $(8,8,8)(8,8,8)$ \\
\hline O3 & $(-1,4,9)(-2,4,10)$ & $(-2,0,2)(-4,0,4)$ & $(-2,0,2)(-4,0,4)$ & $(2,5,8)(2,5,8)$ \\
\hline Demand & $(3,4,5)(3,4,5)$ & $(2,6,10)(1,6,11)$ & $(9,9,9)(9,9,9)$ & $(14,19,24)(13,19,25)$ \\
\hline
\end{tabular}

Now, using the step 5 to the step 7 of the intuitionistic fuzzy zero point method, we have the following reduced intuitionistic fuzzy transportation table.

\begin{tabular}{|c|c|c|c|c|}
\hline & $D_{1}$ & $D_{2}$ & $D_{3}$ & Supply \\
\hline O1 & $(2,7,12)(-3,7,17)$ & $(0,0,0)(0,0,0)$ & $(3,9,15)(2,9,16)$ & $(4,6,8)(3,6,9)$ \\
\hline O2 & $(0,0,0)(0,0,0)$ & $(-1,8,17)(-4,8,20)$ & $(-6,0,6)(-12,0,12)$ & $(8,8,8)(8,8,8)$ \\
\hline O3 & $(-3,5,13)(-7,5,17)$ & $(-2,0,2)(-4,0,4)$ & $(-2,0,2)(-4,0,4)$ & $(2,5,8)(2,5,8)$ \\
\hline Demand & $(3,4,5)(3,4,5)$ & $(2,6,10)(1,6,11)$ & $(9,9,9)(9,9,9)$ & $(14,19,24)(13,19,25)$ \\
\hline
\end{tabular}

Now, using the allotment rules of the intuitionistic fuzzy zero point method, we have the allotment

\begin{tabular}{|c|c|c|c|c|}
\hline & $D_{1}$ & $D_{2}$ & $D_{3}$ & Supply \\
\hline O1 & & $(4,6,8)(3,6,9)$ & & $(4,6,8)(3,6,9)$ \\
\hline O2 & $(3,4,5)(3,4,5)$ & & $(3,4,5)(3,4,5)$ & $(8,8,8)(8,8,8)$ \\
\hline O3 & & $(-6,0,6)(-8,0,8)$ & $(4,5,6)(4,5,6)$ & $(2,5,8)(2,5,8)$ \\
\hline Demand & $(3,4,5)(3,4,5)$ & $(2,6,10)(1,6,11)$ & $(9,9,9)(9,9,9)$ & $(14,19,24)(13,19,25)$ \\
\hline
\end{tabular}

The intuitionistic fuzzy optimal solution in terms of triangular intuitionistic fuzzy numbers: 
$\tilde{x}_{12}^{I}=(4,6,8)(3,6,9), \tilde{x}_{21}^{I}=(3,4,5)(3,4,5), \tilde{x}_{23}^{I}=(3,4,5)(3,4,5) \tilde{x}_{32}^{I}=$ $(-6,0,6)(-8,0,8), \tilde{x}_{33}^{I}=(4,5,6)(4,5,6)$, Hence, the minimum total intuitionistic fuzzy transportation cost is

$\operatorname{Min} \tilde{Z}^{I}=(41,90,139)(21,90,159)$

\section{Results and discussion}

The minimum total intuitionistic fuzzy transportation cost is

$$
\tilde{Z}^{I}=(41,90,139)(21,90,159)
$$

The result in (1) can be explained (refer to Figure 1) as follows

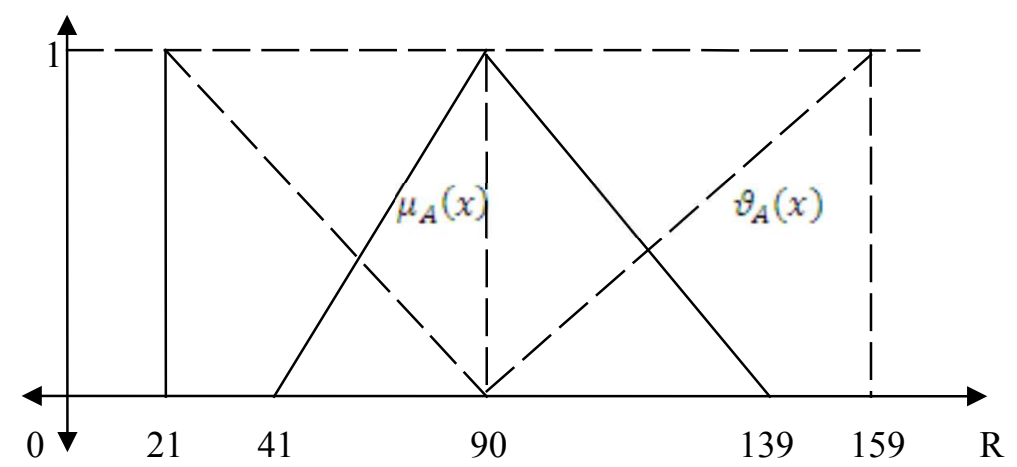

Figure 1: Graphical Representation of IFTC

(a) Transportation cost lies in $[41,139]$.

(b) $100 \%$ expect are in favour that the transportation cost is 90 as $\mu_{\tilde{A}^{I}}(x)=$ $1, x=90$.

(c) Assuming that $\mathrm{m}$ is a membership value and $\mathrm{n}$ is a non-membership value at c. Then $100 \mathrm{~m} \%$ experts are in favour and $100 \mathrm{n} \%$ experts are opposing but $100(1-m-n) \%$ are in confusion that the transportation cost is $c$

Values of $\mu_{\tilde{Z}}(c)$ and $\nu_{\tilde{Z}}(c)$ at different values of c can be determined by using the equations given below:

$$
\mu_{\tilde{Z}}(x)=\left\{\begin{array}{ll}
0 & \text { for } x<41 \\
\frac{(x-41)}{49} & \text { for } 41 \leq x \leq 90 \\
1 & \text { for } x=90 \\
\frac{(139-x)}{49} & \text { for } 90 \leq x \leq 139 \\
0 & \text { for } x>139
\end{array} \quad \nu_{\tilde{Z}}(x)= \begin{cases}1 & \text { for } x<21 \\
\frac{(90-x)}{69} & \text { for } 21 \leq x \leq 90 \\
0 & \text { for } x=90 \\
\frac{(x-90)}{69} & \text { for } 90 \leq x \leq 159 \\
1 & \text { for } x>159\end{cases}\right.
$$




\section{Conclusion}

Mathematical formulation of intuitionistic fuzzy transportation problem and procedure for finding an intuitionistic fuzzy optimal solution for a MIFTP are discussed with relevant numerical example. In this paper new method for proposed to find the optimal solution of MIFBTPs in which supplies, demands and costs are different types of numbers. So, the method can be applied to solve real world transportation problems where the dada is not in symmetric numerical values. The new multiplication operation of triangular intuitionistic fuzzy numbers is employed to get the optimal solution in terms of triangular intuitionistic fuzzy numbers. The proposed method is very simple and easy to understand.

\section{References}

[1] K.T.Atanassov, Intuitionistic fuzzy sets, fuzzy sets and systems, vol.20, no.1.pp.87- 96, (1986), doi: 10.1016/S0165-0114(86)80034-3 .

[2] Amit Kumar, Amarpreet Kaur, Anila Gupta, Fuzzy Linear Programming Approach for Solving Fuzzy Transportation probles with Transshipment, J Math Model Algor (2011) 10:163-180, doi: 10.1007/s10852-010-9147-8.

[3] Annie Varghese and Sunny Kuriakose, Notes on Intuitionistic Fuzzy Sets Vol.18, 2012, No.1, 19-24.

[4] R.Bellman, L.A.Zadeh,Decision making in a fuzzy environment, management sci.17(B)(1970)141-164, doi: 10.1287/mnsc.17.4.B141.

[5] S.Ismail Mohideen, P.Senthil Kumar, A Comparative Study on Transportation Problem in fuzzy environment. International Journal of Mathematics Research, Vol.2 Number.1 (2010), pp. 151-158.

[6] R.Jahir Hussain, P.Senthil Kumar, Algorithmic approach for solving intuitionistic fuzzy transportation problem, Applied Mathematical Sciences, Vol.6, 2012, no. 77-80.

[7] R.Jahir Hussain, P.Senthil Kumar, An Optimal More-for-Less Solution of Mixed Constrains Intuitionistic Fuzzy Transportation Problems, Int.J. Contemp.Math.Sciences, Vol.8, 2013.no.12, 565-576, doi: 10.12988/ijcms. 
[8] R.Jahir Hussain, P.Senthil Kumar, Transportation Problem with the aid of Triangular Intuitionistic Fuzzy Numbers, MMASC 2012 Vol.1 Coimbatore Institute of Technology, Coimbatore.

[9] P.Pandian and G.Natarajan, A new algorithm for finding a fuzzy optimal solution for fuzzy Transportation problems. Applied mathematics sciences, Vol. 4, 2010, no.2, 79-90.

[10] D.Stephen Dinager, K.Palanivel,The Transportation problem in fuzzy environment, int.journal of Algorithm, computing and mathematics, vol2, no3, 2009.

[11] L.A. Zadeh, Fuzzy sets, information and computation, vol.8, pp.338-353, (1965), doi: 10.1016/S0019-9958(65)90241-X. 